\title{
Dano de Caryedes sp. (COLEOPTERA; BRUCHIDAE) e seus Reflexos na Propagação de Enterolobium contortisiliquum (LEGUMINOSAE)
}

\author{
Danilo Barros Donato, Adriano Geraldo Fonseca, \\ Sebastião Lourenço de Assis Júnior, Evandro Luiz Mendonça Machado, \\ Diego Faustolo Alves Bispo \\ Departamento de Engenharia Florestal, Faculdade de Ciências Agrárias, \\ Universidade Federal dos Vales do Jequitinhonha e Mucuri - UFVJM
}

\begin{abstract}
RESUMO
O objetivo deste trabalho foi quantificar o dano de Caryedes sp. em sementes de Enterolobium contortisiliquum; testar diferentes metodologias de superação da dormência; e determinar a influência destes danos na germinação e vigor. As sementes sadias foram submetidas a cinco tratamentos para superação de dormência. Foram avaliados 500 frutos totalizando 5245 sementes, com média de 10,47 $\pm 2,99$ por fruto. Destas, 9,00 $\pm 3,32$ estavam sadias e 0,26 \pm 0,63 danificadas pelo bruquídeo. A escarificação mecânica no sentido do hilo promoveu maior germinação e foi utilizada para comparar com as sementes atacadas. O ataque do bruquídeo pode diminuir significativamente a germinação das sementes de tamboril.
\end{abstract}

Palavras-chave: sementes, Coleoptera Bruchidae, escarificação.

\section{Damage Caused by Caryedes sp. (COLEOPTERA; BRUCHIDAE) and their Reflections in the Propagation of Enterolobium contortisiliquum (LEGUMINOSAE)}

\begin{abstract}
The aim of this study was to quantify the damage caused by Caryedes sp. in seeds of E. contortisiliquum; to test different methods to overcome dormancy; and to determine the influence of this damage on the germination and vigor of the seeds. Healthy seeds were submitted to five treatments in order to overcome dormancy. Five-hundred fruits with a total 5,245 seeds, with a mean of $10.47 \pm 2.99$ per fruit, were evaluated. As a result, $9.00 \pm 3.32$ were considered healthy and $0.26 \pm 0.63$ were damaged by the bruchid. The scarification toward the heel promoted higher germination and it was used for comparison to the attacked seeds. The bruchid attack can significantly reduce the germination of tamboril seeds.
\end{abstract}

Keyword: seeds, Coleoptera Bruchidae, scarification. 


\section{INTRODUÇÃO}

A Família Fabaceae compreende 670 gêneros e cerca de 17.500 espécies, com distribuição cosmopolita. A espécie Enterolobium contortisiliquum (VELL.) Morong. é uma árvore decídua e frondosa que ocorre em florestas pluviais e semidecíduas do norte ao sul do Brasil. Seus frutos podem conter de 2 a 18 sementes, são brilhantes e de coloração marrom. Apresentam-se como vagens recurvadas em formato de rim ou de orelha, o que lhe rendeu diversos nomes populares, como orelha de negro e orelha de macaco. Também é conhecida como tamboril ou timbaúva. Sua madeira é leve, macia, pouco resistente e utilizada para a fabricação de canoas, caixotaria, brinquedos e compensados. É uma espécie pioneira, de rápido crescimento inicial e muito rústica, apropriada para áreas de reflorestamento e recomendada para arborização urbana e recomposição de áreas degradadas (Santos, 1987; Lorenzi, 2002; Carvalho, 2003).

A multiplicação seminal de E. contortisiliquum normalmente é lenta devido ao mecanismo de dormência provocado, provavelmente, pela impermeabilidade do tegumento à água (Lêdo, 1977; Eira et al. 1993). Embora seja um eficiente mecanismo para garantir a perpetuação da espécie, a dormência das sementes é um fator limitante para a produção de mudas, podendo causar desuniformidade na germinação e perda de até $40 \%$ de recursos genéticos se nenhum tratamento de superação for utilizado (Carneiro, 1995). Além disso, pode promover o aumento do risco de perda de sementes por deterioração, já que estas permanecem mais tempo no solo antes da germinação (Eira et al., 1993; Cardoso, 2004). No entanto, a dormência das sementes pode ser superada pela utilização de tratamentos como a escarificação mecânica ou química, alcançando resultados de 70 a $89 \%$ no período de 7 a 14 dias (Durigan \& Nogueira, 1990; Gonzáles, 1994).

Estudos de levantamentos de pragas têm mostrado que as sementes da maioria das leguminosas são severamente danificadas por insetos, especialmente pelos coleópteros. Muitos destes danos promoveram redução acentuada na germinação e vigor das sementes (Loureiro et al., 2004).
Os besouros da família Bruchidae são importantes predadores de sementes, especialmente da família Fabaceae. Têm grande importância econômica por competirem com o homem por grãos, além de afetarem a reprodução de espécies arbóreas e arbustivas (Southgate, 1979). Os adultos são compactos e globulares e apresentam o corpo recoberto por pêlos curtos. Os fêmures das pernas posteriores são espessados e os élitros curtos, deixando exposto o último segmento abdominal, o pigídio (Gallo et al., 2002).

As sementes de tamboril são normalmente atacadas pelos carunchos Caryedes bicoloripes e Merobruchus bicoloripes (Coleoptera: Bruchidae) e pelo Lophopoeum timbouvae (Coleoptera: Cerambycidae), sendo que o ataque conjunto dos dois últimos pode destruir até $50 \%$ das sementes (Santos, 1994; Link \& Costa, 1995). Wink et al. (2007) constataram também o ataque de lagartas Cryptophlebia carpophagoides (Lepidoptera: Olethreutidae). Também é comum o aparecimento de fungos saprófitas ou fitopatogênicos, principalmente como agentes de danos pós-colheita, afetando a qualidade e a quantidade de sementes produzidas (Aguiar et al., 1993).

A escarificação, como método de superação de dormência, é o tratamento que provoca a ruptura ou o enfraquecimento do tegumento, de modo que permita a germinação da semente. A predação de sementes pode ser considerada um processo de escarificação natural auxiliando a germinação (Carvalho \& Nakagawa, 2000), e pode, em alguns casos, ser benéfica, desde que não danifique o embrião e o consumo de substâncias de reserva das sementes seja pequeno, de modo que permita o desenvolvimento da plântula.

Pesquisas voltadas para as essências florestais nativas brasileiras são escassas, principalmente envolvendo as técnicas de superação de dormência e a danificação de sementes por insetos. O objetivo deste trabalho foi quantificar o dano de Caryedes sp. em sementes de Enterolobium contortisiliquum, testar diferentes metodologias de superação da dormência e avaliar a influência destes danos na germinação e vigor. 


\section{MATERIAL E MÉTODOS}

Foram coletados 500 frutos de E. contortisiliquum provenientes de cinco árvores, no mês de outubro de 2008, no município de Gouveia, MG (18 27' 14" S e $\left.43^{\circ} 44^{\prime} 27^{\prime \prime} \mathrm{W}\right)$. Estes foram levados ao Laboratório de Entomologia Florestal da Universidade Federal dos Vales do Jequitinhonha e Mucuri (UFVJM) para identificação da ocorrência e nível de predação nas sementes.

Os frutos foram acondicionados em gaiolas de madeira teladas e com tampa de vidro com dimensões de $30 \times 30 \times 30 \mathrm{~cm}$ e dispostos em sala climatizada com $25 \pm 2{ }^{\circ} \mathrm{C}$, UR $70 \pm 10 \%$ e fotofase de 12 horas, até a emergência dos adultos. Estes foram coletados, mortos em gases tóxicos, montados e enviados para identificação por especialistas.

Os frutos foram abertos $\mathrm{e}$ as sementes quantificadas e classificadas nas categorias indenes, atacadas (por insetos e/ou fungos) e chochas (mal formadas). A porcentagem de substrato consumido foi obtida pela diferença de peso entre as sementes sadias e danificadas pelo bruquídeo, utilizando cinco amostras de 10 sementes de cada categoria.

Após a quantificação de danos provocados pelo bruquídeo, as sementes foram levadas ao laboratório de Sementes Florestais da UFVJM onde foram avaliados o peso de matéria seca e o teor de umidade, as técnicas de superação de dormência, o vigor e a porcentagem de germinação.

Para determinação do teor de umidade e peso de matéria seca, as sementes foram colocadas em estufa $\left(105 \pm 3{ }^{\circ} \mathrm{C}\right)$ por 24 horas, conforme recomendação das Regras para Análise de Sementes (Brasil, 2009).

Para as técnicas de superação de dormência, os seguintes tratamentos foram utilizados:

- T1 - escarificação mecânica lateral com lixa;

- T2 - escarificação mecânica no sentido do hilo com lixa;

- T3 - embebição em água por 72 horas;

- T4 - imersão em água a $100^{\circ} \mathrm{C}$ por 3 minutos;

- T5 - imersão em ácido sulfúrico (100\%) concentrado por 5 minutos; e

- T6 - controle (sem tratamento prévio).

Os tratamentos foram constituídos de quatro repetições com cinquenta sementes cada, distribuídas uniformemente em papel germitest umedecido e levados para germinadora (BOD) em temperatura de $25 \pm 2{ }^{\circ} \mathrm{C}$, com fotoperíodo de 12 horas. $\mathrm{O}$ melhor tratamento obtido para as sementes sadias foi utilizado para comparar com o percentual de germinação de sementes atacadas, sendo as sadias usadas como controle. Este ensaio constou de quatro repetições com 25 sementes cada.

$\mathrm{O}$ vigor e a porcentagem de germinação por tratamento foram monitorados durante 21 dias, considerando como sementes germinadas aquelas com emissão de radícula.

Os valores da porcentagem da germinação foram transformados em $\operatorname{arcsen} \sqrt{\frac{\mathrm{x}}{100}}$ e as médias, submetidas à análise de variância e comparadas pelo teste de Tukey ao nível de $5 \%$ de significância (Santana \& Ranal, 2004).

\section{RESULTADOS E DISCUSSÃO}

Foram quantificadas 5244 sementes, com média de 10,47 $\pm 2,99$ por fruto. Destas, 4508 estavam aptas para a reprodução (indenes), representando $85,95 \%$ das sementes. As demais, provavelmente, não germinarão, pois $593(11,31 \%)$ estavam mal formadas (chochas) e $15(0,29 \%)$ apresentavam-se com fungos. O bruquídeo danificou 128 sementes representando 2,45\% das sementes (Figura 1).

O peso médio de uma semente sadia é de $0,82 \mathrm{~g}$. $\mathrm{O}$ ataque dos bruquídeos reduziu o peso para $0,58 \mathrm{~g}$. Por diferença, obtém-se o substrato consumido por um besouro que foi de 0,24 g (Figura 2), correspondendo a uma perda de $28,96 \%$ da semente.

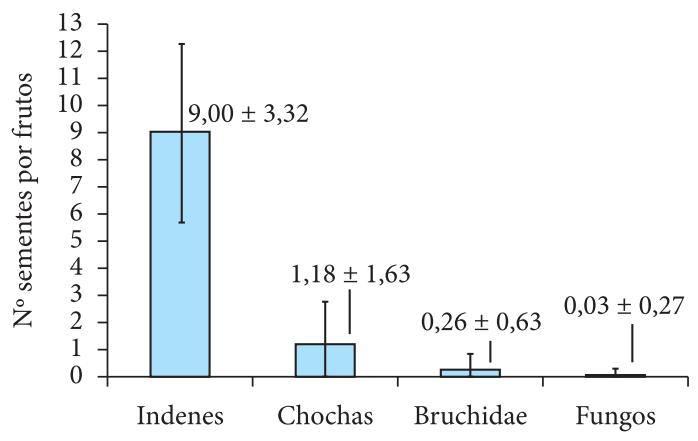

Figura 1. Sementes de E. contortisiliquum indenes, chochas e atacadas por Coleoptera Bruchidae e fungos. Figure 1. Seeds of E. contortisiliquum healthy, aborted, and attacked by Coleoptera Bruchidae and fungi. 
Embora essa quantidade de substrato alimentar, necessária para completar o ciclo, seja maior em Caryedes sp., seus danos são menos severos que Caryedes bicoloripes, que consome $21,48 \%$ do peso, pois este chega a danificar $18,90 \%$ das sementes (Santos, 1994), ao passo que o primeiro danifica, apenas, 2,45\% das sementes (Figura 1).

A escarificação mecânica com lixa no sentido do hilo foi o método mais efetivo para a superação da dormência em sementes de E. contortisiliquum com 94,5\% de sucesso, seguida da escarificação lateral com $83,5 \%$ de germinação. A imersão em água no ponto de ebulição por 3 minutos e o tratamento químico com ácido sulfúrico $\left(\mathrm{H}_{2} \mathrm{SO}_{4}\right)$ concentrado por 5 minutos não diferiram entre si, sendo superiores à embebição em água por 72 horas e ao controle (sem tratamento prévio). Esses dois últimos não apresentaram diferença significativa e foram os que tiveram menor porcentagem de germinação, com $6 \%$ e $5 \%$, respectivamente (Tabela 1 ).

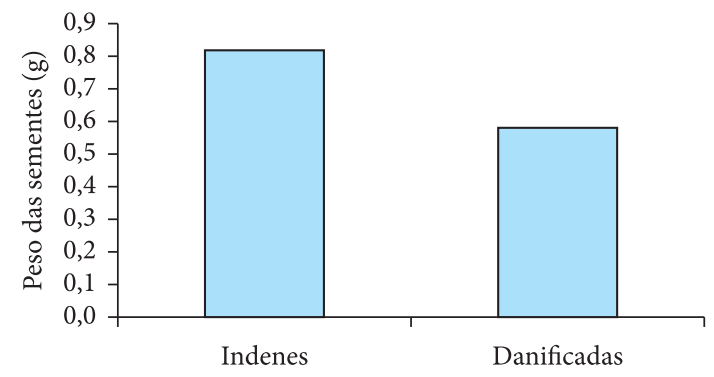

Figura 2. Substrato alimentar consumido por Caryedes sp. (Coleoptera: Bruchidae) em sementes de E. contortisiliquum.

Figure 2. Feed substrate consumed by Caryedes sp. (Coleoptera: Bruchidae) in seeds of E. contortisiliquum.
Valores em uma mesma coluna seguidos por uma mesma letra não diferem entre si pelo teste de Tukey a $5 \%$ de probabilidade.

O tratamento envolvendo escarificação mecânica da semente também proporcionou a maior porcentagem de germinação em sementes de E. contortisiliquum (Alexandre et al. 2009) e E. schomburgkii (Rigamonte-Azevedo et al., 2007). Por outro lado, encontram-se nos estudos de Lêdo (1977), Eira et al. (1993) e Fowler \& Bianchetti (2000) que o tratamento mais recomendado para superação de dormência em sementes de E. contortisiliquum é a escarificação química, envolvendo a imersão em $\mathrm{H}_{2} \mathrm{SO}_{4}(75 \%)$ por 30 minutos.

O tratamento mais efetivo na superação de dormência de sementes de tamboril, no presente estudo (escarificação mecânica no sentido do hilo com lixa) foi utilizado para comparar com as sementes atacadas pelo bruquídeo, as quais alcançaram uma porcentagem de germinação de $5 \%$ (Tabela 2).

Esta baixa porcentagem de germinação e vigor mostra que o ataque do Caryedes sp. tem influência significativa na germinação das sementes de tamboril, provavelmente pelo alto consumo de substrato da semente.

\section{CONCLUSÕES}

A danificação da semente pelo bruquídeo e por fungos, aliada às sementes mal formadas afeta de forma negativa a produção de mudas do tamboril.

Tabela 1. Porcentagem média do vigor e da germinação em diferentes tratamentos de superação de dormência em sementes indenes de E. contortisiliquum.

Table 1. Mean percentage of germination and vigor in different treatments for breaking dormancy in healthy seeds of E. contortisiliquum.

\begin{tabular}{lcc}
\multicolumn{1}{c}{ Tratamentos } & \multicolumn{2}{c}{ Médias } \\
\cline { 2 - 3 } & Vigor & Germinação \\
1. Escarificação no sentido do hilo & $97,0 \pm 2,6^{\mathrm{a}}$ & $94,5 \pm 4,1^{\mathrm{a}}$ \\
2. Escarificação lateral & $85,5 \pm 5,5^{\mathrm{b}}$ & $83,5 \pm 5,5^{\mathrm{b}}$ \\
3. Água $100^{\circ} \mathrm{C}$ & $36,0 \pm 6,3^{\mathrm{c}}$ & $62,5 \pm 8,7^{\mathrm{c}}$ \\
4. $\mathrm{H}_{2} \mathrm{SO}_{4}$ & $23,5 \pm 8,5^{\mathrm{c}}$ & $34,5 \pm 9,8^{\mathrm{d}}$ \\
5. Embebição 72 horas & $4,5 \pm 2,5^{\mathrm{d}}$ & $6,0 \pm 2,8^{\mathrm{e}}$ \\
6. Controle & $3,0 \pm 2,0^{\mathrm{d}}$ & $5,0 \pm 1,6^{\mathrm{e}}$ \\
\hline
\end{tabular}


Tabela 2. Porcentagem média do vigor e da germinação de sementes de E. contortisiliquum, indenes e atacadas por Caryedes sp.

Table 2. Mean percentage of vigor and germination of of E. contortisiliquum healthy and attacked seeds by Caryedes sp.

\begin{tabular}{lcc}
\multicolumn{1}{c}{ Tratamentos } & Vigor (\%) & Germinação (\%) \\
1. Escarificação mecânica em semente indene & $91,0 \pm 7,57^{\mathrm{a}}$ & $91,0 \pm 7,57^{\mathrm{a}}$ \\
2. Semente atacada & $5,0 \pm 2,00^{\mathrm{b}}$ & $5,0 \pm 2,00^{\mathrm{b}}$ \\
3. Controle (sem tratamento prévio) & $1,0 \pm 2,00^{\mathrm{b}}$ & $3,0 \pm 3,83^{\mathrm{b}}$ \\
\hline
\end{tabular}

As sementes de tamboril responderam diferentemente aos tratamentos, apresentando maior velocidade de emergência quando escarificadas no sentido do hilo.

Os danos provocados pelos bruquídeos podem ser considerados um fator positivo por superar a dormência. Seu consumo de substrato pode ter sido responsável pela baixa porcentagem de germinação das sementes de tamboril.

\section{AGRADECIMENTOS}

À Professora Dra. Cibele Stramare Ribeiro Costa da UFPR pela identificação do gênero do bruquídeo.

\section{STATUS DA SUBMISSÃO}

Recebido: $16 / 07 / 2010$

\section{AUTOR(ES) PARA CORRESPONDÊNCIA}

\section{Sebastião Lourenço de Assis Júnior}

Departamento de Engenharia Florestal,

Faculdade de Ciências Agrárias,

Universidade Federal dos Vales do Jequitinhonha e Mucuri - UFVJM, Rod. MG 367, Km 583, 5000, Alto da Jacuba, CEP 39100-000,

Diamantina, MG, Brasil

e-mail: assisjr@ufvjm.edu.br

\section{REFERÊNCIAS}

Aguiar IB, Piña-Rodrigues FCM, Figliolia MB. Sementes florestais tropicais. Brasília: Associação Brasileira de Tecnologia de Sementes; 1993.

Alexandre RS, Gonçalves FG, Rocha AP, Arruda MP, Lemes EQ. Tratamentos físicos e químicos na superação de dormência em sementes de Enterolobium contortisiliquum (Vell.) Morong. Revista Brasileira de Ciências Agrárias 2009; 4(2):156-159.

Brasil. Ministério da Agricultura. Regras para analise de sementes. Brasília: DNDV/ CLAV; 2009. 365 p.

Cardoso VJM. Dormência estabelecimento do processo. In: Ferreira AG, Borghetti F. (Orgs.) Germinação: do Básico ao aplicado. Porto Alegre: Artmed; 2004. p. 95108.

Carneiro JGA. Produção e controle de qualidade de mudas florestais. Curitiba: UFPR/FUPEF; Campos : UENF; 1995. $451 \mathrm{p}$.

Carvalho NM, Nakagawa J. Sementes: ciência, tecnologia e produção. Jaboticabal: Funep; 2000. 588 p.

Carvalho PER. Espécies arbóreas brasileiras. Brasília: EMBRAPA Informação tecnológica; 2003. 1039 p.

Durigan G, Nogueira JCB. Recomposição de matas ciliares. São Paulo: Instituto Florestal; 1990. 14 p. Série Registro, n. 4.

Eira MTS, Freitas RWA, Mello CMC. Superação da dormência de sementes de Enterolobium contortisiliquum (VELL.) Morong. Leguminosae. Revista Brasileira de Sementes 1993; 15:177-181.

Fowler AJP, Bianchetti A. Dormência em sementes florestais. Colombo: Embrapa Florestas; 2000. 27 p. Documentos, n. 40.

Gallo D, Nakano O, Silveira Neto S, Carvalho RPL, Batista GC, Berti Filho E et al. Entomologia agrícola. Piracicaba: FEALQ; 2002.920 p.

Gonzáles AE. Evaluación de la dinâmica del crescimiento primário para cuatro espécies forestales nativas em plantaciones de enriquecimento em bosques subtropicales de Argentina. Eldorado 1994; 5:99-104.

Lêdo AAM. Estudo da dormência em semente de Guapuruvú (Schizolobium parahyba (Vell.) Blake) e Orelha de Negro (Enterolobium contortisiliquum (Vell.) Morong) e métodos para sua quebra [tese]. Viçosa: Universidade Federal de Viçosa; 1977.

Link D, Costa EC. Danos causados por insetos em sementes de timbaúva, Enterolobium contortisiliquum (Vell.) Morong. Ciência Florestal 1995; 5(1):113-122. 
Lorenzi H. Árvores brasileiras: manual de identificação e cultivo de plantas arbóreas nativas do Brasil. Nova Odessa: Plantarum; 2002. 188 p.

Loureiro MB, Carvalho AG, Rossetto CAV. Danos causados por insetos na germinação e no vigor de sementes de Apuleia leiocarpa Vog. Macbride. Agronomia 2004; 38(1):105-109.

Rigamonte-Azevedo V, Almeida MC, Firmino JL. Germinação de sementes de timbaúba (Enterolobium schomburgkii Benth)- Mimosoidae. In: Anais do VIII Congresso De Ecologia Do Brasil; 2007; Caxambu. Caxambú: Sociedade de Ecologia do Brazil; 2007. 2 p.

Santana DG, Ranal MA. Análise da Germinação: um enfoque estatístico. Brasília:Editora Universidade de Brasília; 2004. 248 p.
Santos E. Nossas madeiras. Belo Horizonte: Editora Itatiaia; $1987.313 \mathrm{p}$.

Santos GP, Anjos N, Zanuncio JC, Alves JB. Danos por Cariedes bicoloripes (PIC) (Coleoptera: Bruchidae) e Lophopoeum timbouvae LAMEERE (Coleoptera: Cerambycidae) em frutificações de tamboril, Enterolobium contortisiliquum Leguminosae. Ciência e Prática 1994; 18:104-108.

Southgate BJ. Biology of the Bruchidae. Annual Review of Entomology 1979; 24:449-473. doi:10.1146/annurev. en.24.010179.002313

Wink C, Guedes JVC, Murari AB, Pelentir SCS. Ocorrência de Cryptophlebia carpophagoides Clarke, 1951 (Lepidoptera: Olethreutidae) em frutos de timbaúva, Enterolobium contortisiliquum (Vell.) Morong, no estado do Rio Grande do Sul, Brasil. Ciência Florestal 2007; 17(1):7-79. 